$100 \mathrm{~s}$. The agreement of amplitude and rate shows that the slow phase in the recovery of activity is rate limited by the isomerization of proline 93, and argues for refolding taking place only from chains in which that proline is in the native cis form.

Lin and Brandts have now studied two more of the four proline residues in ribonuclease. Proline 117 is most probably completely trans in both native and unfolded states and therefore will make no contribution towards kinetic complexity. On the other hand proline 114 is completely cis in the native protein and 50 per cent cis in the reversibly unfolded state, which correlates with the lowamplitude slowest refolding phase. This residue must be in the cis configuration for the protein to regain activity ${ }^{9}$.

The major fastest phase of the refolding kinetics is thus unaccounted for and the isomerization of proline 42 has yet to be investigated - the experiments will be slow and tricky. While Lin and Brandts are willing to acknowledge the existence of the non-native active intermediate their view is that, if it accounts for the major refolding phase, it is unlikely to involve proline isomerization. In support of this, they quote unpublished work of Fisher et al. This group has purified a new enzyme, peptidylprolyl-cis, trans-isomerase ${ }^{10}$, which catalyses the kinetic steps associated (by Lin and Brandts) with isomerization of prolines 93 and 114 but has no effect on the major fast phase. Does this enzyme require the ribonuclease to be unfolded in order to catalyse the isomeri-

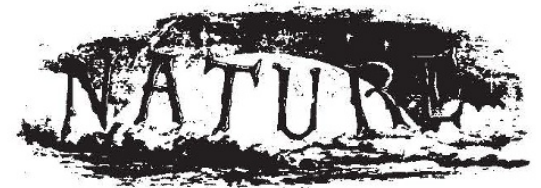

\section{0 years ago}

AN excellent opportunity of observing the aerial means of propulsion in the flying fish was afforded me during a six days' calm lately when crossing the Bay of Bengal. This must be my excuse for again touching this subject. I watched day by day some hundreds rise under the bows of the ship. The water surface was a glassy calm. As each fish rose it spread its wings at once, apparently beating the surface with them two or three strokes before they steadied out. The tail which, of course, under water was in rapid motion, to escape from the ship, now gave ten or a dozen rapid beats, which could be counted by the ripples on the still surface, and the fish was off in aerial flight. As each fish lost the impetus of the first rise, which generally happened at about forty yards, the binoculars showed us the anal fins, which had till now been fully extended, drooping to feel the water. As soon as the surface was felt the tail was quickly introduced, and five or six smart strokes, aiso indicated by ripples, brought the impetus up again and carried the fish about another thirty yards, when another droop sent it on again, and so forth, some of the older fish travelling in this way 400 to 500 yards.

From Nature 32 147, 18 June 1885. zation of proline, offering support for the Brandts model? To add to the confusion, Schmid, Buonocore and Baldwin ${ }^{11}$ have not only further confirmed their sequential model but have also strengthened the evidence that the major phase shows the characteristics of proline isomerization.

On a slightly different tack, a further advance in assigning kinetic phases to distinct regions of the ribonuclease structure has come with the discovery by Labhardt that the S-peptide and S-protein fragments of RNase $S$ can be distinguished by circular dichroism ${ }^{12}$. From the kinetics of conformational changes of both the Speptide and the S-protein during their association and refolding reactions, Labhardt comes out firmly in support of a sequential type of folding pathway. Intriguingly, his experiments show that the Sprotein folds (to a form as yet uncharacterized) in a fast phase before the S-peptide folds to its native helical structure. This fast phase corresponds to 65 per cent of the total change, greater than the proportion of fast folding species $U_{F}$ in scheme 2 . The folded form must correspond in part at least to $\mathrm{I}_{1}$.

Since Labhardt has already shown that recombination between S-peptide and Sprotein occurs fast and very early in the folding pathway of RNase $S^{13}$, we have to envisage, contrary to most previous expectations, that the association between these two regions occurs at a stage when the S-peptide, and possibly the S-protein, possess no native-like conformation. This would be in keeping with the poorly hydrogen-bonded associated species detected by $\mathrm{NMR}^{14}$. If Labhardt's results, obtained with a relatively 'simple' system, are of general application, the model of 'flickering clusters' of secondary and super-secondary structure, which associate to be stabilized in the native conformation, will have to be considerably revised.

The circular dichroism results have also raised a problem relating to the unfolding kinetics. The unfolding rates for RNase $S$ and S-protein, which have been followed by monitoring changes specifically in the S-protein moiety, parallel the formation of the slowly refolding species $\mathrm{U}_{\mathrm{S}}$ which contains non-native proline isomers (scheme 2). The random isomerization of proline to form $\mathrm{U}_{\mathrm{S}}$ had previously been thought to occur in the spectroscopically 'silent' reaction $\left(\mathrm{U}_{\mathrm{F}} \rightleftharpoons \mathrm{U}_{\mathrm{S}}\right)$ but Labhardt's results suggests that, in terms of the model of scheme 2 , there must be a rate-limiting proline isomerization $\mathrm{I}_{2} \rightarrow \mathrm{I}_{1}{ }^{\prime}$ which is faster than $\mathrm{I}_{2} \rightarrow \mathrm{U}_{\mathrm{F}}{ }^{\prime}$, followed by a rapid conformational unfolding $1_{2} \rightarrow U_{s}$. Alternatively, the rate determining step is a conformational unfolding $\mathrm{I}_{2} \rightarrow \mathrm{U}_{\mathrm{F}}{ }^{\prime}$ followed by a rapid isomerization to $U_{S}$. Neither alternative fits easily with the expected relative rates of these processes.

The frustrating aspect of the whole question of proline isomerization is that, however the issue is resolved, it will tell us nothing about the actual mechanism of protein folding. Though it is becoming possible to see some of the detailed processes at work in the folding of ribonuclease, the methods have not yet overcome the kinetic madness of the system.

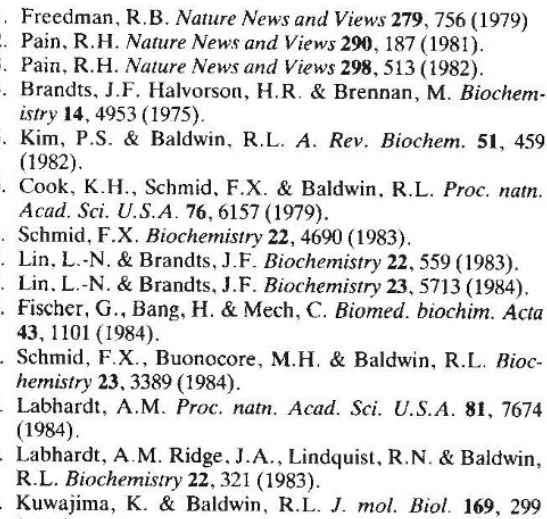
(1983).

Roger Pain is Professor of Biochemistry, The University, Newcastle upon Tyne NEI 7RU, UK.

\title{
Astronomical spectroscopy
}

\section{Interstellar space contains the largest encountered atoms}

from William D. Watson

OBSERVATIONS published on page 647 of this issue ${ }^{1}$, in conjunction with recent Soviet publications ${ }^{2,3}$ highlight the presence of extremely high Rydberg states in which the outer electron of an atom has been excited to very high energies - in the interstellar medium. These results confirm and significantly extend recent identifications ${ }^{4,5}$ of spectral lines at radio frequencies an order of magnitude lower than those at which lines had previously been detected. Discoveries of new lines at radio wavelengths have until now tended ○) 1985 Nature Publishing Group to be associated with the high-frequency (millimetre and submillimetre) end of the spectrum.

Because of the appearance of several lines in the low-frequency series, it is clear that they result from radiative transitions involving extremely high Rydberg states (or radio-recombination lines) and changes of one unit in the principal quantum number of the state (alpha transitions). The lowest frequency at which these lines have been detected corresponds to the 732 alpha transition, which 